\title{
Current status and future prospect of surgical treatment for pancreatic cancer
}

\author{
Yinmo Yang \\ Department of General Surgery, Peking University First Hospital, Beijing 100034, China \\ Correspondence to: Yinmo Yang. Department of General Surgery, Peking University First Hospital, Beijing 100034, China. Email: yangyinmo@263.net.
}

Submitted Nov 26, 2019. Accepted for publication Dec 04, 2019.

doi: 10.21037/hbsn.2019.12.04

View this article at: http://dx.doi.org/10.21037/hbsn.2019.12.04

Pancreatic cancer is one of the most deadly malignancies, and the incidence of this dismal disease has been reported to rise yearly in a global range. Currently, the cancerrelated mortality rate for pancreatic cancer ranks the fourth in western countries. However, it is predicted that by 2030 , the cancer-related death rate of pancreatic cancer will rise to the second place in the United States, and the third place in Europe (1). The main reason for this change is that the treatment efficacy of top-ranking cancers, such as breast, prostate and colorectal cancer, has accomplished a significant improvement recently, while the progress in pancreatic cancer treatment is still evolving slowly. Situations of the diagnosis, treatment and prognosis of pancreatic cancer in China are similar. Overall, the treatment of pancreatic cancer is still extremely challenging, and radical resection remains the only curative therapy. Although some progress has been achieved recently in both adjuvant and neoadjuvant therapies, there is still a long way to go to improve the long-term prognosis of patients with pancreatic cancer. In this mini-review we summarize current status focusing on major advancements in surgical treatment of pancreatic cancer during the past 20 years, and highlights future prospects in the field.

\section{Tremendous improvements in surgical techniques have been achieved despite incomplete understanding of molecular mechanism of pancreatic cancer}

The past two decades have witnessed a great improvement on surgical treatment for pancreatic cancer at the technical level. The improvement of surgical techniques is associated with an increasing resection rate and a remarkable decline of perioperative mortality and the incidence of severe complications after surgery. Some of modern oncological principles, for example, the refine of resection margin status, the standardized extent of regional lymphadenectomy, and the concepts of artery-first approach and removal of the periarterial nerve plexus, have been proved to increase the local radicality and reduce the local recurrence rate. Moreover, the concept and technology of minimally invasive surgery have been increasingly popular. In particular, laparoscopic and robotic pancreatic surgery are replacing almost all types of open surgeries for the treatment of pancreatic cancer. However, these advancements in technology did not bring significant improvement in the long-term prognosis of pancreatic cancer patients. The majority of patients after a radical surgery will develop local or systemic recurrence, resulting in median overall survival of 28-54 months. Compared with breast cancer, lung cancer, colorectal cancer, and other common cancers, our knowledge of biological behavior of pancreatic cancer is still limited, and there is still a long way to go to develop effective targeting drugs against pancreatic cancer for clinical applications.

\section{The transformation of the treatment paradigm of pancreatic cancer: from "Surgery First" to "Multiple Disciplinary Team"}

"Surgery First" was the prior standardized treatment paradigm of pancreatic cancer. Under this paradigm, we emphasized the advantages of a specific specialty but turned a blind eye to the difference of the same disease in different stages. This concept might cause over-treatment or undertreatment, and need update. Currently, the treatment of pancreatic cancer has shifted from traditional specialtycentered "Surgery First" paradigm to disease-centered "Multiple Disciplinary Team" paradigm. 


\section{From adjuvant chemotherapy to neoadjuvant chemotherapy: a prior shift of chemotherapy}

Post-operative adjuvant chemotherapy is the standardized treatment for pancreatic cancer and should be conducted routinely. While some topics on adjuvant therapy remain hot such as the details of the regimen of adjuvant therapy, recent focus has been shifted to the efficacy and necessity of neoadjuvant therapy as a major adjustment to treatment strategy. The National Comprehensive Cancer Network (NCCN) Guideline of the United States used to provide treatment options of neo-adjuvant chemotherapy or upfront surgery for patients with borderline resectable pancreatic cancer. Since 2016, However, treatment strategy for borderline-resectable pancreatic cancer has been to revised to neoadjuvant chemotherapy only in NCCN Guideline. Furthermore, neo-adjuvant chemotherapy is recommended in patients with resectable pancreatic cancer with high recurrent risks, such as serum CA-199 level exceeding 500 $\mathrm{U} / \mathrm{mL}$, large bulk, and suspected lymph node metastasis (2). The idea of comprehensive treatment with a "prior-shift of chemotherapy" is reflected by the application the neoadjuvant chemotherapy. Recent evidences with high quality and quantity have supported the potential of neoadjuvant chemotherapy in improving the prognosis of pancreatic cancer patients, highlighting the trend in cancer therapy.

\section{Personalized precision medicine: from morphological to molecular typing}

The traditional clinical and histological typing of pancreatic cancer is based on morphology and imaging presentations with limited guidance in a clinical setting. The same treatment has been provided for different subtypes of diseases, without distinguishing individual differences in clinical behaviors such as relapse, metastasis, and sensitivity to radiation and chemotherapy from the perspective of tumor biology. In addition, personalized treatment is not emphasized which reflects the precision medicine principle of "treating individual respectively even for the same disease." With the ongoing development of highthroughput sequencing technology and systemic biology, the traditional typing of pancreatic cancer is transformed to a more precise one at molecular level, laying the basis for individualized targeted therapy. Our current pancreatic cancer molecular typing based on the next-generation sequencing and proteomics technology is still at primary stage with limited clinical guidance. Typing disparities in the number of types and detailed categorization can be secondary to the differences in the size and source of the samples, and methodology selection in the studies, which may indicate the molecular and pathological characteristics of pancreatic cancer. Due to the peculiarity and complexity of the biological behaviors of pancreatic cancer, there is still a large gap in our comprehensive interpretation of the molecular biological behaviors of pancreatic cancer (3). Recently, Golan et al. conducted a prospective phase 3 trial called POLO (Pancreas Cancer Olaparib Ongoing) to evaluate the efficacy of olaparib as maintenance therapy in patients who had a germline BRCA mutation and metastatic pancreatic cancer after first-line platinum-based chemotherapy (4) A significant difference in progressionfree survival was observed in trial group compared to control group. The POLO study serves as a milestone for the first precision medicine treatment model in that the targeted therapy is based on the biomarker of an individual gene.

In summary, the 20-year history of diagnosis and treatment of pancreatic cancer has shown the dramatic transition from empirical treatment to the individualized regimen, from limit-exploring surgery to perfection-pursuing operation, and from anatomical resection to biotherapy. Recent breakthroughs in the diagnosis and treatment of breast cancer, colorectal cancer, and liver cancer will facilitate the transition of pancreatic cancer diagnosis from anatomical typing based on morphology to the molecular typing based on precision medicine. Consequently, surgeons are required to re-shape their mindset to pursue individualized and targeted therapy for pancreatic cancer to achieve better outcomes in patients with this lethal disease.

\section{Acknowledgments}

None.

\section{Footnote}

Conflicts of Interest: The author has no conflicts of interest to declare.

\section{References}

1. Rahib L, Smith BD, Aizenberg R, et al. Projecting cancer incidence and deaths to 2030: the unexpected burden of thyroid, liver, and pancreas cancers in the United States. Cancer Res 2014;74:2913-21.

2. National Comprehensive Cancer Network. (NCCN) 
Clinical Practice Guidelines in Oncology. Pancreatic Adenocarcinoma, Version 3.2019, Available online: https:// www.nccn.org/professionals/physician_gls/default.aspx. Accessed November 27, 2019.

3. Waddell N, Pajic M, Patch AM, et al. Whole genomes

Cite this article as: Yang Y. Current status and future prospect of surgical treatment for pancreatic cancer. HepatoBiliary Surg Nutr 2020;9(1):89-91. doi: 10.21037/hbsn.2019.12.04 redefine the mutational landscape of pancreatic cancer. Nature 2015;518:495-501.

4. Golan T, Hammel P, Reni M, et al. Maintenance Olaparib for Germline BRCA-Mutated Metastatic Pancreatic Cancer. N Engl J Med 2019;381:317-27. 